\title{
LA FAMILIA EN SU HORA MÁS CRÍTICA. ASPECTOS CUESTIONABLES SOBRE DISFUNCIONALIDAD Y ORIGEN DE LA PERVERSIÓN.
}

\author{
FAMILY FACING ITS MOST CRITICAL TIME. QUESTIONABLE \\ ASPECTS OF ITS DYSFUNCTIONALITY AND THE ORIGIN OF \\ PERVERSION.
}

Elvis Jorge Alcalde Muñoz ${ }^{1}$

\section{Resumen}

El presente artículo, brinda un panorama sobre la familia, la disfuncionalidad y aspectos referidos al fenómeno de la conducta desviada, a la vez que enfatiza sobre el rol del Estado en políticas punitivas y preventivas haciendo un paralelo al respecto. De otro lado, pretende fomentar una verdadera mecánica de políticas sociales preventivas en torno a la familia como núcleo de la sociedad y que sea el principal motor de cambio de nuestra realidad criminógena.

Palabras claves: Familia, matrimonio, hijos, controles sociales informales, disfuncionalidad, violencia familiar, neurosis, psicopatía, conducta desviada, criminógeno, políticas preventivas.

\begin{abstract}
This paper provides an overview of the institution of family, its dysfunctionality, and aspects related to the deviant behavior phenomenon while emphasizing on the role of the State in drafting punitive and preventive policies. On the other hand, the paper aims to promote a true mechanism for preventive social policies regarding family as the nucleus of society and as the main driver of change in our criminogenic context.
\end{abstract}

Keywords: Family, Marriage, Children, Informal Social Controls, Dysfunctionality, Domestic Violence, Neurosis, Psychopathy, Deviant Behavior, Criminogenic Context, Preventive Policies.

Doctor en Derecho y Ciencia Política (UNMSM); Mágister en Derecho - Ciencias Penales (UNMSM); Consultor - Calificador del Consejo Nacional de la Magistratura y Docente de la Escuela de Post-Grado de la Universidad Femenina del Sagrado Corazón. Dirección electrónica: alcalde_662@hotmail.com 
SUMARIO: I. Introducción; II. La Justicia penal, control social insuficiente; III. Familia disfuncional, psicopatías, parafilias y eventos neuróticos; IV. Avances en punición y políticas preventivas; V. Conclusiones; VI. Referencias Bibliográficas.

\section{INTRODUCCIÓN}

La familia como entidad esencial dentro de una sociedad ha ido adquiriendo distintas variantes y tendencias en cuanto a su conceptualización, en ese aspecto la idea de núcleo fundamental ligado a los lazos meramente de sangre se ha ido modificando y ampliando hasta adquirir la idea de "familia grande" que actualmente domina el mundo contemporáneo. Estos aspectos se ven reflejados en cuanto hoy, una persona puede crecer y desarrollarse en torno a modelos familiares no solamente vinculados a un padre o madre biológico, sino que puede interrelacionarse y seguir patrones de conducta de una madre sustituta, de un padre adoptivo, o de terceros que en buena cuenta también son considerados parte de la entidad familiar. Lo importante es que la familia debe mantener una funcionalidad y estabilidad, capaz de adaptarse a los cambios no sólo de sus integrantes, sino de la sociedad en la que se desenvuelve ${ }^{2}$.

El mundo occidentaly moderno presenta cambios constantes de paradigmas y patrones de conducta, así también la idea misma de constitución familiar ha ido variando con el desarrollo de la propia mujer dentro de la sociedad; así, hoy en día, ella ocupa y se desenvuelve en iguales condiciones que el hombre, se capacita y asume cargos importantes dentro de la empresa estatal y privada lo que le ha valido niveles de independencia y desarrollo significativos. En general, debido a un mundo cada vez más moderno, competitivo y veloz, la mayor parte de los integrantes de un núcleo familiar adquieren roles y expectativas de vida y desarrollo profesional cada más exigentes, no conformándose con lo justo y necesario, lo que en alguna medida colateralmente afectaría también la interrelación familiar por los altos niveles de ausencia del hogar.

Otro aspecto a resaltar, no menos importante, lo constituye la formalidad de la pareja familiar, que para nuestra sociedad lo constituye el matrimonio, institución que ha venido deteriorándose en la última década, así las estadísticas como en el caso peruano ${ }^{3}$ reflejan el alto índice de separaciones y divorcios, lo que significa una voluntad por formalizarse pero la problemática se manifiesta en la poca duración de estos enlaces.

En ese sentido, en relación a los cambios y adaptación de la familia en el mundo occidental, Vid. Gaitán, Lourdes (2006) Sociología de la Infancia- Nuevas Perspectivas. Madrid: Edit Síntesis; p. 144.

Tendencia en alza, Fuente: Instituto Nacional de Estadística e Informática - INEI: La cantidad de parejas que opta por el divorcio va en aumento pues de 5,625 procesos en 2011, ha variado a 13,873 procesos en noviembre 2015 . 
Actualmente, el matrimonio no es para toda la vida, sino hasta donde dure. En esa línea el matrimonio como institución familiar enfrenta un problema que tendría una explicación variante, v.gr. deficiencia educacional, y una pobre educación psico-sexual y en valores, aunado a modelos inadecuados que generan inestabilidad emocional al momento de elegir la pareja. No debemos dejar de lado también como posible factor el fenómeno de la independencia y la poca tolerancia debido precisamente a la abrumadora competitividad existente en todas las esferas de la vida.

En esta medida, los cambios a la interna del grupo familiar son claves y repercuten en la formación de los individuos que se educan, crecen y se desarrollan dentro de él. Si bien la persona no adopta patrones de conducta por herencia genética, sin embargo si adopta conductas positivas o negativas, ya desde la infancia, dependiendo de los modelos que observa. Así, la figura materna y paterna es clave en la evolución del individuo, por ello también se debate actualmente sobre el valor legal que se ha dado a las uniones de hecho entre parejas del mismo sexo (v.gr. en Holanda, Bélgica, Canadá, España y Massachusset-EEUU entre otros), cuestionando algunos sectores sobre los derechos que estas uniones asumirían frente a funciones familiares propiamente dichas como la adopción y crianza de hijos.

La familia de hoy es una institución atomizada, moderna y con integrantes independientes, buscando mejorar su propia vida, con ideales de felicidad cada vez más exigentes, en que los niveles de tolerancia son escasos y la competitividad es exagerada, de ahí que nos toca adaptarnos a una sociedad convulsionada y vertiginosa, y aprovechar lo positivo de un mundo moderno, post-contemporáneo para procurar interrelaciones familiares sanas y beneficiosa no sólo para los hijos, sino para la totalidad de sus integrantes.

\section{LA JUSTICIA PENAL, CONTROL SOCIAL INSUFICIENTE}

Sin duda, el hombre, por naturaleza, es un ser conflictivo, en soledad o en comunidad, crea problemas, está en una constante lucha por el dinero, el amor, el sexo, la justicia, así está lleno de pasiones, bondades y maldades. En esa línea, en sociedad, los conflictos que crea tienen en alguna medida que ser prevenidos (ex ante) y reprimidos (ex post), así el derecho penal como medio de control social formal ${ }^{4}$

\footnotetext{
Al respecto, sobre el Derecho Penal como control penal: "Los instrumentos de los cuales se vale el Derecho Penal para la protección de los bienes jurídicos suelen ser más severos que otras ramas del ordenamiento jurídico. Por lo que la utilización de dichos mecanismos sólo ha de ser posible cuando la sociedad no puede controlar graves conflictos. Siendo uno de esos recursos estatales: la pena. Pero esta necesidad no basta para que la pena sea autorizada, sino que ésta debe ser proporcional y deberá encuadrarse dentro de un ámbito legal garantista. Esta amarga necesidad que constituye la pena por las consecuencias que conlleva para el individuo hace, que sólo se recurra a ella como ultima ratio, es decir, el último recurso a emplear por no existir otros medios más eficaces" (Peña Cabrera, Raúl (1995) Tratado Derecho Penal-Estudio Programático de la Parte General- Tomo I. Lima: Editorial GRIJLEY E.I.R.L, 2da Edición, p. 129).
} 
buscará controlar la conducta desviada, pero como último recurso y cuando ya se ha producido el daño. A través de los jueces y las autoridades del sistema judicial de un país, el Estado ejerce esta potestad sancionadora que en el fondo es un tipo de violencia legitimada dentro de un marco normativo que lo faculta.

El XVII Congreso Mundial de Criminología llevado a cabo en el año 2014 en la ciudad de Monterrey, México, ha puesto como temática eje de debate público penal a la violencia, agresión y delincuencia juvenil a través de las pandillas, así como señalando a la inseguridad ciudadana o poblacional como un fenómeno regional. Situación que se ha convertido en una problemática generalizada de la mayor parte de países latinoamericanos, y de la que no es ajeno el Perú, no debemos olvidar que el rostro de la criminalidad de nuestro país es juvenil, y por el cual nosotros hemos venido adoptando políticas de control de ultima ratio, es decir el Estado ha asumido su rol monopólico de ejercicio coercitivo, más que preventivo. Así, nuestras legislaciones presentan sanciones penales elevadas para sancionar y negar el desvalor de la acción que ocasionan los delitos en las calles, pero no debemos olvidar que el alto índice de castigos penales que se aplica a la criminalidad por violaciones sexuales, homicidio, sicariato, robos, tráfico ilícito de sustancias controladas, entre otros, representan en el fondo una especie de fracaso como sociedad, ya que reconocemos que la incidencia criminal está aumentando, necesitamos abrir más centros carcelarios para que los delincuentes cumplan sus penas, existe hacinamiento que no es posible manejar. Podrá seguir aumentando el presupuesto para construir más centros carcelarios, hasta cuándo. Esta forma de Control Formal siempre será necesaria en una sociedad moderna y democrática, sin embargo se debe tender a disminuir su uso, pero para ello debe efectivizarse los demás controles "informales" como la familia, escuela, el deporte, la educación. Definitivamente, ante mayor eficiencia del control informal, menor debe de ser el uso del control formal.

Dentro de los controles informales, la familia cumple un rol trascendental en la formación del individuo, sin duda su defensa en los diferentes ordenamientos del mundo es importante para su vigencia y estabilidad. Hoy, el fenómeno de la violencia familiar atenta contra la estabilidad familiar porque la destruye, no desde fuera, sino desde y por dentro. La violencia es uno de los factores de desintegración y destrucción familiar y por la cual los hijos se quedarán sin modelos adecuados a seguir, consecuentemente este control social fracasaría. Por ello, el papel del Estado en la consolidación de la familia con políticas preventivas sociales es fundamental, así los programas sociales de capacitación y guía no sólo deben estar orientados a la niñez, sino a 
toda la institución familiar. La base de la formación se encuentra en la familia, el menor aprende por modelos de conducta, por guía e imitación, valores que son adquiridos en el hogar, para luego consolidarse en la escuela y la sociedad.

\section{FAMILIA DISFUNCIONAL, PSICOPATÍAS, PARAFILIAS Y EVENTOS NEURÓTICOS}

Mucho se ha hablado del fenómeno de la familia disfuncional, y si bien representa un problema del cual derivan otros, sin embargo definirlo constituye un verdadero cajón de sastre ya que al hablar de una disfuncionalidad del núcleo familiar nos referimos a hogares rotos, incompletos, deshechos, pero también carenciales, caóticos, inseguros, tiránicos y/o violentos, en buena cuenta se ha roto el funcionamiento normal y socialmente aceptable de este grupo de personas ligados por un lazo intimo incuestionable. Esta ruptura perjudica y es nociva para sus integrantes a pronto, mediano o largo plazo, así producto de estas familias se forman individuos con diversas trastornos de índole psicológicos y hasta psiquiátricos. Una de las familias más nocivas es la carencial ${ }^{5}$ debido a que en esta modalidad existe abandono ya sea físico o emocional y en la cual la ausencia de la madre conlleva una carga emocional mucho más fuerte para los hijos y que si no es subsanada y guiada adecuadamente, puede generar adultos conflictivos en el futuro. Este tópico ha sido objeto de diversos estudios por investigadores como Erik ERIKSON (1950), D. W. WINNICOTT (1967), John BOWLBY (1968, 1973 y 1980) que identifican la ausencia materna como decisiva en la formación de los hijos menores.

No podemos negar que en todas las formas de disfuncionalidad, existe un perjuicio para el desarrollo de los integrantes del seno familiar, en la cual muchas veces adoptan trastornos psicológicos que si no son tratados oportunamente pueden derivar en desviaciones neuróticas, psicóticas, psicopáticas y/o parafílicas y que desencadenen actos desviados o delictivos contra la sociedad. Ya se sabe que las neurosis son de diversos tipos ${ }^{6}$ presentando sintomatología variada como excesiva ansiedad, depresión, síntomas histéricos y obsesivos compulsivos, pero la relevancia en cuanto a su consecuencia criminógena la vemos con mayor frecuencia en las neurosis obsesivas compulsivas y la histeria. Así, la neurosis se manifiesta en la persona producto de una fuerte actividad represiva del recuerdo de malas experiencias sobre todo infantiles, dentro del hogar.

En esa línea, sobre familias carenciales como factor criminógeno: Vid. Solís Espinoza, Alejandro (2004) Criminología - Panorama Contemporáneo. Lima: Edit ByB; p. 288.

$6 \quad$ Al respecto, sobre las diversas modalidades de neurosis, Vid. Sanz de la Garza, Joaquim Homs (1996) Trastorno Mental Transitorio y Drogas que inciden en la Imputabilidad. Barcelona: José María Bosch Editor S.L.; pp. 148 y 149. 
En buena cuenta, el evento neurótico está íntimamente ligado al regreso (desde el inconsciente) de esa mala experiencia no superada o no tratada adecuadamente en el pasado, por lo que dicho hecho se reaviva en la persona con el recuerdo produciendo angustia, ansiedad y generando de inmediato la conducta compulsiva impostergable (tics nerviosos, movimientos bruscos, sudoración fría, espasmos, contracciones, etc.) que es un problema no sólo para la persona que lo sufre sino también para su entorno. Aquí vemos las conductas imprudentes que cometen sobre todo los responsables de realizar un trabajo fino y delicado como los médicos cirujanos, los cirujanos plásticos, los constructores, etc. que requieren de una concentración especial y que se ve alterada por la llegada de la conducta compulsiva y que ocasiona falencia en su trabajo. Estos casos son procesados como ilícitos imprudentes.

Definitivamente, todas las neurosis están acompañadas de un motor de decepción, frustración, ansiedad y finalmente conducta "anormal" exteriorizada, por ello, si en la neurosis obsesiva compulsiva el recuerdo ocasiona angustia, por el contrario el componente más resaltante en la histeria es la frustración y la poca tolerancia ante ello, por lo que aquí el afán excesivo de figuración y ser el centro de atención es fundamental, así el histérico sufre cuando no es atendido, pero el origen de este foco histérico también está en el pasado sobre todo cuando la persona ha crecido en medio de innumerables carencias y decepciones que no han sido canalizadas adecuadamente por un adulto guía. Lo que se debe entender es que las neurosis, en general, se van haciendo con el pasar de la vida, teniendo como foco de origen la niñez, la adolescencia y se exterioriza ya de adulto, pero se esconde los síntomas generalmente por vergüenza; por ello, no es advertida por la gente que lo rodea, salvo por el entorno más cercano e íntimo; es más, puede existir incluso profesionales con estos trastornos y que muchas veces aplacan la sintomatología entregándose excesivamente al trabajo. En el caso del brote histérico se puede manifestar en conductas desviadas como injurias, difamaciones, daños a la propiedad u otros ilícitos dolosos producto del afán exacerbado de ser atendido o llamar la atención.

Los estudios sobre el comportamiento violento y agresiones han arrojado que v.gr. los pederastas (parafilia) o abusadores sexuales de menores ${ }^{7}$ han tenido una infancia llena de carencias donde no estuvo presente la madre (carencia física o emocional), así también los individuos que adolecen de trastorno disocial de personalidad (psicopatía) han experimentado violencia

Al respecto, sobre las diversas formas de actuación del pederasta: Ortiz Valero, Tomás y Ladrón de Guevara y Guerreo, Javier (1998) Lecciones de Psiquiatría Forense. Granada: Editorial Comares S.L; p. 332. 
y agresividad durante su formación, en esa línea el común denominador en todas estas personas es la desorganización y desestructuración de su entorno familiar. Debemos tener presente que la neurología está estudiando algunas desviaciones mentales y en las parafilias como en la psicopatía se ha señalado medicamente la existencia de focos orgánicos cerebrales alterados que contribuirían a la aparición del trastorno, así en el caso de los pederastas, el hipotálamo cerebral tendría una función decisiva en la alteración de la conducta sexual. Sin embargo, a este nivel, si bien este órgano regula los instintos primarios como son la sed, el calor, el frio, el deseo sexual, explicándose en una sobrecarga sexual, la medicación aplacaría el problema, pero la elección equivocada del objeto sexual deseado se mantendría vigente, por lo que el fármaco no haría efecto en esenivel de decisión. Así, el tratamiento farmacológico sería apropiado para mantener niveles moderados de deseo sexual pero tendría que ser complementado con la terapia psicológica para obtener respuestas positivas.

No negamos el avance de la ciencia médica y las teorías que se puedan esbozar en torno a la aparición de las trastornos mentales, pero por el momento el factor social es decisivo en su inicio, salvo aquellas que ya se ha corroborado fehacientemente una fuente genética como es el caso de la psicosis (esquizofrenias). Las psicopatías hoy conocidas como trastorno disocial de personalidad son trastornos realmente graves que la medicina neurológica también ha tratado de darle un fundamento orgánico cerebral señalando la falencia de la zona prefrontal, explicando en el sentido de un mal funcionamiento de la células de esa parte de masa cerebral y que sería el origen del comportamiento malsano y de poco o nulo aprecio por los semejantes, la manipulación y el impulso a dañar a los demás. Sin embargo, creemos que si bien estas estructuras pueden provenir dañadas desde el nacimiento o producto de un accidente, el entorno social y de modelos es fundamental para que la persona pueda realizar conductas desviadas en el futuro y ocasionar daño a sus semejantes. Cuestión polémica aquí constituye el hecho de que la ciencia médica ha esbozado incluso alguna posibilidad de intervenciones quirúrgicas en la zona prefrontal para este tipo de personas psicopáticas, teniendo en cuenta que precisamente esta zona es la que deja menos secuela física o motora de toda la masa cerebral.

Recordemos, que estas desviaciones, para nosotros tienen un origen decisivo en la formación del individuo y el entorno que lo rodea, dentro de ello la familia - factor social, (salvo el evento psicótico que tiene fundamento genético), ocasionan conductas desviadas de gravedad, así los ilícitos penales más feroces y que conmocionan a la sociedad son cometidos por estas personas, v.gr. violaciones sexuales de menores de edad, asesinatos 
en serie - serial killers, asesinatos en masa, secuestros, sicariato, etc. El caso de las neurosis merece una mención aparte del grupo precitado porque son trastornos mentales más leves que tienen diversa sintomatología y que el interés de la criminología se restringe a las modalidades obsesivocompulsivas y la histeria que también tiene incidencia criminal pero cuando la enfermedad está bastante pronunciada.
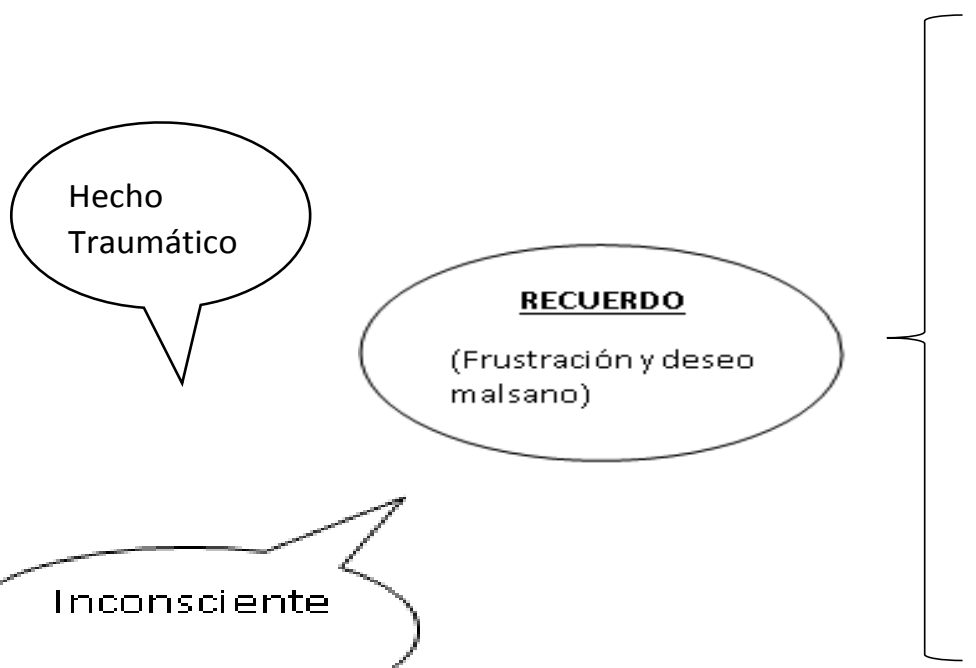

Neurosis
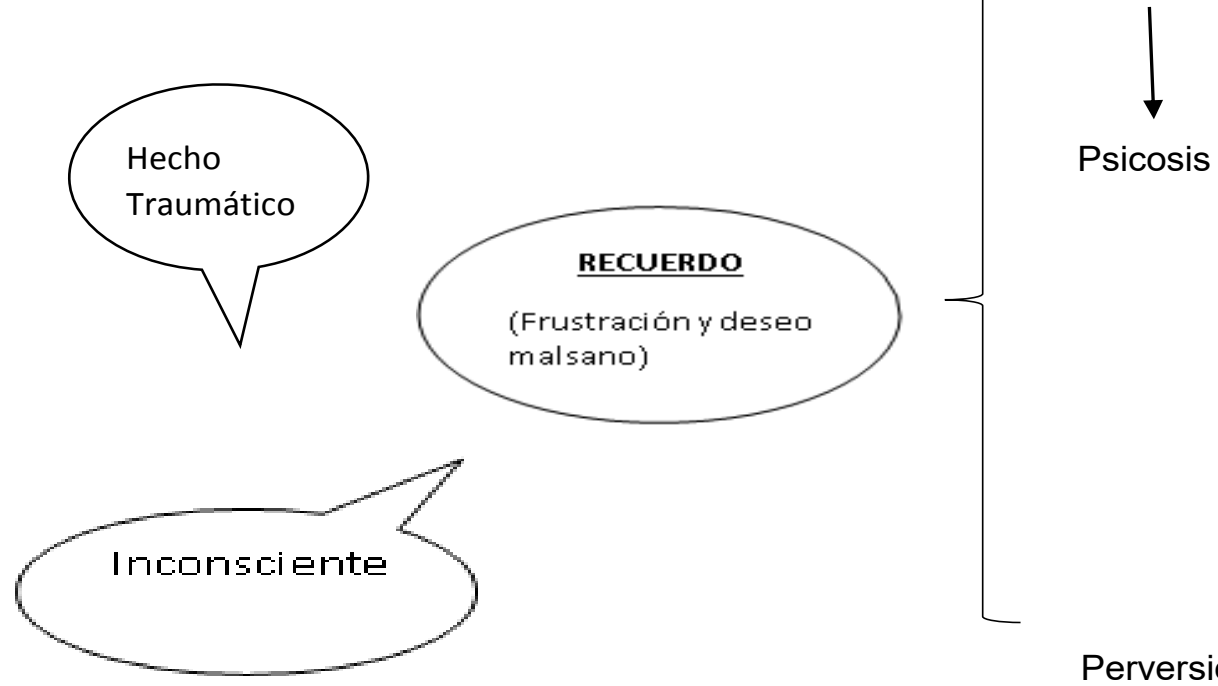

Perversión

\section{Gráfico $N^{\circ} 1$}

El brote psicótico, es considerado actualmente como uno de los trastornos psiquiátricos más severos ${ }^{8}$, que en buena cuenta tiene un origen genético, sin embargo, al ser un trastorno eminentemente juvenil, su aparición está ligada a la etapa de la adolescencia y juventud, todavía cuando la persona está bajo un fuerte influjo familiar. Si bien la primera alucinación visual o auditiva causa alerta, el papel del entorno más cercano es clave en el manejo de la sintomatología por lo cual la tolerancia y paciencia con el paciente es fundamental para una evolución favorable. Si bien la farmacología es decisiva, también lo es el apoyo familiar. Es frecuente observar en países de esta parte de la región como los condenados a medida de seguridad por delitos cometidos bajo el influjo de la esquizofrenia paranoide muchas veces son abandonados por su entorno más cercano, ello puede obedecer a múltiples factores, entre los cuales estaría lo complejo de la enfermedad y

Sobre las principales características y sentimientos que aquejan a los pacientes de esquizofrenia paranoide, Vid. Goldman, Howard H.(1992) Psiquiatría General. México: Edit. Manual Moderno S.A. de C.V ; pp. 256 y 257 (Traducido por Lic. Hortencia Beatriz Vera López). 
la dificultad de tratar a este tipo de pacientes, así como la negativa, muchas veces de éstos a tomar la medicación.

Los antipsicóticos han tratado de inhibir y regular las sustancias neurotransmisoras cerebrales entendiéndose que ahí estaría el foco psicótico que genera el desfase con la realidad producto de una sobre actividad de estas sustancias, sin embargo constituye un trastorno que no desaparece del todo por lo que se requeriría tratamiento permanente para controlar la alucinación y paranoia con la que va íntimamente aparejada. De otro lado, el psicoanálisis ha esbozado una explicación distinta a la médica sobre el origen de la psicosis, así ha señalado, que éste trastorno es un grado más avanzado de la neurosis, explicando en principio que la perversión es la foto en negativo de la neurosis, así mientras que en la perversión la persona se entrega a sus bajos deseos por el contrario en la neurosis existe represión y fuerte rechazo de los fuertes deseos. Pero, el punto de quiebre se da cuando estos deseos malsanos son intensos que el individuo ya no puede reprimirlos adecuadamente entonces se da la ruptura del mundo psíquico, pasando del evento neurótico al brote psicótico. Esto debido al esfuerzo psíquico que significa contrarrestar el deseo malsano. Como lo vemos en el Grafico $\mathrm{N}^{\circ}$ 1, el rompimiento del psiquismo y el paso de la neurosis al brote psicótico equivalen a una especie de escape de una realidad nociva que nos quiere dominar.

Al ser el evento neurótico el precedente del brote psicótico, definitivamente, el psicoanálisis ha tratado de explicar una problemática que va de menos a más, producto precisamente de un factor social en que la persona no ha podido superar sus traumas surgidos en las primeras etapas de la vida. No podemos negar que actualmente la farmacología ha entrado a tallar casi en todas los tratamientos para enfermedades mentales. En el caso psicótico, los antipsicóticos han logrado controlar los periodos de alucinación y paranoia, evitando que sean extensos, precisamente en la etapa más agresiva de la enfermedad como lo es la esquizofrenia paranoide donde se produce la mayor parte de conductas desviadas y/o ilícitos penales.

\section{AVANCES EN PUNICIÓN Y POLÍTICAS PREVENTIVAS}

En cuanto a la sanción, el Estado peruano ha ido realizando algunas modificaciones a la legislación penal sobre todo agravando las penas en cuanto a ciertos ilícitos donde haya existido un vínculo de parentesco entre autor y víctima, entendiendo que la relación parental que une a personas dentro del seno familiar es especial y debe albergar altos valores y principios éticos a respetar. Así, lo que buscamos como sociedad a través de la normatividad 
vigente es fortalecer y procurar la vigencia de la institución familiar, evitar su deterioro y destrucción, es por ello que se desvalora con mayor intensidad la acción y el resultado de un crimen donde el autor es el hijo y la víctima el padre, que si el autor y víctima fueran dos amigos. De otro lado, también se ha empezado a dar importancia al tratamiento de la violencia familiar, así, algunas teorías propugnaban que el Estado no debía entrar a tallar dentro de los problemas a la interna del hogar, entendiendo que deberían solucionarse sólo con la intervención de sus integrantes, tal es así que incluso en muchas legislaciones no lo tenemos como un delito a la "violencia familiar" sino como circunstancia que cualifica como agravante de otras figuras delictivas.

Sin embargo, actualmente, se ha entendido que si bien la violencia familiar es una situación que se genera dentro del hogar, no menos cierto es que su solución para el mantenimiento de la armonía familiar requiere de ayuda externa y en muchos casos de instituciones tutelares de justicia, ya que la agresión puede ser el inicio de una cadena que acabe en delitos que sí están tipificados por la ley penal como lesiones graves u homicidios (parricidio y/o feminicidio). En esa línea, no debemos dejar de mencionar la dependencia psicológica y el círculo de la violencia que aqueja a la víctima de violencia familiar, lo que hace difícil desligarse del entorno violento, aunado a la presencia de hijos menores dentro del hogar. Se debe precisar que las políticas punitivas (alza de penas) son las que más se trabajan por parte de las autoridades del sector pertinente, las mismas que obedecen a determinada realidad estadística medible, en buena cuenta son de corto plazo, inmediatas y porque no decirlo son fácilmente identificables por la población; así también, ante la ola de crímenes por encargo (llámese sicariato), se incluyó estas figuras como agravantes de otros tipos penales; igual ha ocurrido en su momento con la alta incidencia del feminicidio, la violencia familiar, los abusos sexuales, etc. De manera general, ante una realidad medible de criminalidad, se opta por pedido de facultades legislativas y se eleva las sanciones penales es por ello que el Perú tiene uno de los Código Penales más modificados del continente, pero no obedece a un tratamiento sistemático, a una coherencia en la técnica legislativa; por ello, existen incoherencias, tanto así que delitos cuyos bienes jurídicos son de menor valor tienen penas más altas que aquellos cuyos bienes jurídicos son de mayor valor como la vida o la libertad personal.

No es menos cierto que los políticos o las autoridades que ejercen el poder son poco proclives a implantar verdaderas políticas sociales preventivas; es decir, invertir en capacitaciones, programas de ayuda y fortalecimiento de la familia, educación, deporte, y salud mental ya que los resultados de estas políticas no van a ser inmediatos y no son palpables objetivamente por la población, mientras que la construcción de la carretera, el puente, 
los edificios y el alza de penas si puede ser observado con mayor rapidez y verificado; pero realmente, la criminalidad y la conducta desviada sólo va ir disminuyendo en la medida que se inicie un trabajo preventivo a nivel de los controles sociales informales. Es verdad que tomará su tiempo ver los resultados, pero el hombre desviado sale de un hogar destruido, carencial y sin valores, y es ahí donde se debe trabajar. Mientras no se vire hacia un trabajo con este objetivo, seguiremos construyendo más cárceles.

\section{CONCLUSIONES}

A continuación, señalaremos algunos puntos centrales desarrollados en el presente artículo:

a) El mundo post contemporáneo nos presenta estándares altos de competitividad en todas las esferas del quehacer humano, lo que nos hace ser cada vez más independientes y actuar con mayor eficacia y rapidez;por ello, la familia como núcleo de la sociedad debe ser capaz de adaptarse a estos cambios, no negando la realidad, sino enfrentándola y sacar los aspectos positivos para su bienestar y consolidación.

b) Los últimos Congresos mundiales sobre Criminología y Derecho penal han puesto en el debate la temática de la inseguridad ciudadana, la violencia y la delincuencia juvenil como un problema global, recomendando la implementación de políticas preventivas y de revaloración de la familia como institución fundamental en la formación y educación del individuo.

c) La disfuncionalidad no es un concepto individual o unitario, sino que abarca una serie de falencias y desordenes en el interior del hogar que incluye el tipo de hogar carencial, tiránico, violento, nocivo, entre otros que ocasionan un deterioro permanente de las relaciones intrafamiliares donde sus integrantes sufren y quedan afectados con traumas que si no son tratados adecuadamente desencadenarán adultos conflictivos y propensos a realizar actos desviados en perjuicio de la sociedad.

d) Es importante mantener los niveles de punición; sin embargo, el objetivo de toda sociedad civilizada y moderna es reducir los niveles de criminalidad a su mínima expresión, y para ello se debe implementar oportunamente las políticas preventivas sociales, poniendo especial interés en el fortalecimiento de la familia y su consolidación, evitando su desintegración y desvaloración. 


\section{REFERENCIAS}

Abramowitz, Jonathan S. (2007) Trastorno Obsesivo- Compulsivo. México D.F:Editorial El Manual Moderno S.A. de C.V. (Título Original: Obsessive - compulsive disorder, 2006; traducido por Dra. Martha Elena Araiza Martínez).

Corsi, Jorge. (2004) Violencia Familiar - una mirada interdisciplinaria sobre un grave problema social. Buenos Aires: Editorial Paidós.

Freud, Sigmund. (1985) Compendio de Psicoanálisis. Madrid: Editorial Tecnos S.A. (Título Original: Abriss der Psychoanalyse, redactado en 1938 y publicado por Int Z. Psychoanal en 1940; traducido por Luis López Ballesteros y de Torres).

Gaitán, Lourdes (2006) Sociología de la Infancia- Nuevas Perspectivas. Madrid: Editorial Síntesis S.A.

Goldman, Howard H. (1993) Psiquiatría General, 3era edición, México D.F.: Editorial Manual Moderno S.A. de C.V., (Título Original: Review of General Psychiatry, 1992. Traducción de la 3era edición por Lic. Hortencia Beatriz Vera López - Profesora Univ. Nac. Autónoma de México).

Ortiz Valero, Tomas y Ladrón de Guevara y Guerrero, Javier (1998) Lecciones de Psiquiatría Forense. Granada: Editorial Comares S.L.

Peña Cabrera, Raúl (1995) Tratado de Derecho Penal I - Estudio Programático de la Parte General. Lima: Editorial Grijley E.I.R.L., 2da Edición.

Sanz de la Garza, Joaquín Homs (1996) Trastorno Mental Transitorio y Drogas que inciden en la imputabilidad. Barcelona: José María Bosch Editores S.L.

Solís Espinoza Alejandro (2004) Criminología - Panorama Contemporáneo. Lima: Editores ByB, 4ta edición.

Fecha de recepción : 21 de julio de 2016

Fecha de aceptación : 05 de setiembre de 2016 